\title{
Role of hnRNP A1 in Coronavirus RNA Synthesis
}

\author{
XUMING ZHANG, CHRISTOPHER LYLE, YICHENG WANG, AND LIN \\ ZENG \\ Department of Microbiology and Immunology, University of Arkansas for Medical Sciences, \\ Little Rock, Arkansas 72205, USA
}

\section{INTRODUCTION}

Several host proteins have been identified that bind to the cis-acting sequences of mouse hepatitis virus (MHV) RNAs (Furuya and Lai, 1993; Yu and Leibowitz, 1995; Zhang and Lai, 1995; Li et al., 1997, 1999). The first among those is the heterogeneous nuclear ribonucleoprotein ( $\mathrm{hnRNP}$ ) Al. In normal mammalian cells, hnRNP-A1 is predominantly localized in the nucleus, but it shuttles between the nucleus and the cytoplasm (Dreyfuss et al., 1993). It interacts with a number of RNAs, particularly pre-mRNA and snRNAs, and a number of proteins such as itself, other hnRNPs and some serine-arginine (SR)-rich proteins through its amino terminal RNA-binding domains and the carboxyl terminal glycine-rich domain, respectively, and thus is associated with the RNP complex (Dreyfuss et al. 1993; Buvoli et al., 1988; Cartegni et al., 1996). hnRNP A1 has been implicated in promoting RNA-reannealing (Buvoli et al., 1992) and in regulating alternative RNAsplicing (Dreyfuss et al., 1993). Its involvement in regulation of viral RNA processing of human immunodeficiency virus (HIV) and human T-cell leukemia virus (HTLV)-2 has also been suggested (Black et al., 1995, 1996). Recent evidence indicates that hnRNP Al is required for inhibition of HIV-1 pre-mRNA splicing (Caputi et al., 1999). Previously, we found that hnRNP Al specifically binds to the cis-acting sequences (the negative-strand leader and intergenic [IG] sequence) of MHV RNA (Furuya and Lai, 1993; Zhang and Lai, 1995). Site-directed mutagenesis of the IG sequence indicated that 
the extent of mRNA transcription correlates with the efficiency of hnRNP Al-binding to the IG sequence (Zhang and Lai, 1995). In MHV-infected cells, hnRNP Al is translocated from the nucleus to the cytoplasm ( $\mathrm{Li}$ et al., 1997). These findings suggest that hnRNP Al may play a role in regulation of MHV RNA replication and transcription. However, to date, no direct evidence for a functional role of hnRNP Al in MHV RNA synthesis has been reported.

In this study, we employed a dominant-negative approach by expressing mutant hnRNP Al proteins in cells and then determining the effects of the mutants on viral replication. We found that the mutant hnRNP-Al proteins indeed exerted inhibitory effects on viral cytopathogenicity, cell fusion, viral growth and viral RNA synthesis. Thus, this study provides the direct in vivo evidence demonstrating a functional role for hnRNP Al in MHV RNA synthesis, establishing that hnRNP-Al is a bona fide host factor in regulation of MHV RNA synthesis.

\section{MATERIALS AND METHODS}

\subsection{Virus, Cells and Antibodies}

MHV strain JHM (Makino and Lai, 1989) was used exclusively throughout this study. The murine astrocytoma cell line DBT (Hirano et al., 1974) was used for virus growth, infection and cell lysate preparation. A monoclonal antibody specific to the glycine-rich domain of hnRNP-Al was kindly provided by Dr. Gideon Dreyfuss (University of Pennsylvania). The monoclonal antibody M2 specific to the FLAG was purchased from Strategene (San Diego, CA).

\subsection{Construction of Plasmids}

A cDNA clone of the full-length murine hnRNP Al gene was previously constructed in the pBluescript vector (Stratagene), termed pBSmA1 (Zhang et al., 1999). For expression of the wild-type and mutant A1 proteins, pBS-mA1 DNA was used as a template for PCR amplification with a pair of primers specific for each construct. For the wild-type Al, $\triangle \mathrm{IX}$, $\triangle \mathrm{M} 9$, and $2 \times R B D$, the 5'-sense primer is 5'-mA1-ORF (5'-TTT GGA TCC ATG TCT AAG TCC GAG TC-3'), which contains a Bam HI site (underlined) at the 5 '-end and the first $17 \mathrm{nt}$ of the open reading frame (ORF). The antisense 3'-primers were 3'-mAl-ORF (5'-GAA CCT CCT GCC AC-3') for Al, 3'-mAl-VIII (5'-TTG GTT CCG TGG TTT-3') for 
$\triangle \mathrm{IX}, 3^{\prime}$-mA1-RBDII (5'-GCG ACC TCT CTG ACT-3') for 2xRBD, and 3'mAl- $\triangle \mathrm{M} 9$ (5'-GTT GTA ATT GCC-3') for $\triangle \mathrm{M} 9$. All 3'-primers contain an additional 12-nt sequence (5'-TTT GAA TTC TTA-3) which includes an Eco RI site (underlined) and a stop codon, and a sequence encoding the 8 amino acids flag (5'-CTT GTC ATC GTC GTC CTT GTA GTC-3'). PCR was performed in a DNAsys Thermocycler (M.J. Researches Inc.) for 25 cycles under the following conditions: $95^{\circ} \mathrm{C}$ for $30 \mathrm{sec} .56^{\circ} \mathrm{C}$ for $1 \mathrm{~min}$ and $72{ }^{\circ} \mathrm{C}$ for $1 \mathrm{~min}$. The PCR products were digested with Bam HI and bluntended with T4 DNA polymerase and then digested with EcoRI. The digested PCR fragments were cloned into the EcoRV and EcoRI sites of DI vector $\mathrm{p} 25 \mathrm{CAT}$ to replace the CAT gene, generating pDE-A1, pDE-A1- $\mathrm{IIX}$, pDE-A1- $\triangle \mathrm{M} 9$, and pDE-A1-2xRBD, respectively. For expression of hnRNP A1 proteins by the recombinant vaccinia virus, the first ORF in the DI was removed by digesting the DNAs of pDE-Al and mutants with SnaBI and SpeI. The smaller SnaBI-SpeI fragments $(1.5 \mathrm{~kb}$, representing the DI ORF) were eliminated by agarose gel electrophoresis, while the large SnaBISpeI fragments were excised from the gel and purified by the gel elution kit (Qiagen). The purified DNA fragments were blunt-ended with T4 DNA polymerase and self-ligated with T4 DNA ligase. The resultant constructs pT7-A1, pT7-A1- $\Delta \mathrm{IX}, \mathrm{pT} 7-\mathrm{Al}-\Delta \mathrm{M} 9$, and pT7-A1-2xRBD contain a T7 RNA polymerase promoter, a 20 -nt sequence derived from the DI leader and a 15-nt from the intergenic sequence upstream of the ORF.

\subsection{In Vitro Transcription, Transfection, and CAT Assay}

Plasmid DNAs were linearized with $X b a \mathrm{I}$ and DI RNAs were transcribed in vitro with the MegaScript kit (Ambion Inc.). RNA and DNA transfection was carried out with the DOTAP transfection kit (Boehringer Mannheim). Cell lysates were prepared at $8 \mathrm{~h}$ post-transfection and subjected to CAT assay with the procedure as described previously (Zhang et al., 1994).

\subsection{Radiolabeling of Proteins and Immunoprecipitation}

For detection of the protein expression, cells were starved in methionine-free medium for half hour and labeled with ${ }^{35} \mathrm{~S}$-methionine at $100 \mu \mathrm{Ci} / \mathrm{ml}$ (Amersham-Pharmacia-Biotech) in the presence of Actinomycin $\mathrm{D}$ for $3 \mathrm{hrs}$. Cells were then lysed with a radioimmunoprecipitation assay (RIPA) buffer (50 mM Tris, pH7.4, $150 \mathrm{mM} \mathrm{NaCl}, 0.5 \% \mathrm{NP}-40,0.1 \%$ SDS, $1 \mathrm{mM}$ PMSF). Immunoprecipitation of the lysate was carried out on a rocking platform at $4{ }^{\circ} \mathrm{C}$ for $2 \mathrm{~h}$ to overnight with the M2 anti-FLAG Mab (Stratagene). The antibody-antigen complexes were then precipitated with 
protein A-agarose beads at $4{ }^{\circ} \mathrm{C}$ for $2-4 \mathrm{~h}$. Agarose beads were washed 3 times with RIPA buffer. Proteins complexes were denatured by boiling for 3 min in Lammeli's sample loading buffer (100 mM Tris, pH6.8, $200 \mathrm{mM}$ DTT, 4\% SDS, $0.2 \%$ bromophenol blue, $20 \%$ glycerol) and analyzed by sodium dodecyl sulfate-polyacrylamide gel electrophoresis (SDS-PAGE). The gels were dried and exposed to X-ray film and autoradiographed.

\subsection{Radiolabeling and Analysis of Intracellular Viral RNAs}

Cells were infected with $\mathrm{MHV}$ at an m.o.i. of 1. Intracellular viral RNAs were metabolically labeled with ${ }^{3} \mathrm{H}$-uridine at $100 \mu \mathrm{Ci} / \mathrm{ml}$ (Amersham-Pharmacia- BRL-Gibco) in the presence of actinomycin D $(10 \mu \mathrm{g} / \mathrm{ml})$ at $4 \mathrm{~h}$ post infection for $4 \mathrm{~h}$, and isolated with the Trizol reagent (Gibco-BRL). One $\mu \mathrm{l}$ of RNAs of each sample (from a total of $5 \times 10^{6}$ cells) were precipitated by $5 \%$ trichloroacetic acid (TCA). The incorporation of ${ }^{3} \mathrm{H}$-uridine into the TCA-precipitated materials on the fiberglass filters was then determined by a liquid scintillation counter (Beckman 2000). Radiolabeled RNAs were also denatured with $6 \mathrm{M}$ glyoxyl, and analyzed by electrophoresis on $1 \%$ agarose gel. Gels were treated with Amplifier (Amersham-Phamacia-BRL-Gibco), dried and exposed to X-ray film.

\section{RESULTS AND DISCUSSION}

\subsection{Expression of Mutant A1 Proteins by an MHV DI Expression System}

In the defective-interfering RNA expression system, the gene of interest is placed under the control of an MHV-specific promoter (IG sequence); expression of the inserted gene depends on the presence of helper MHV functions and the transcription of a subgenomic mRNA containing the inserted gene. Here, we used this system for expression of mutant A1 proteins in MHV-infected cells. Because this homologous expression system is devoid of interference by foreign sequences, any effects of the expressed gene on MHV or DI RNA synthesis can be directly assessed. Cells were infected with MHV and transfected with DI RNAs containing the mutant gene. At $4 \mathrm{~h}$ post transfection, intracellular proteins were labeled with ${ }^{35} \mathrm{~S}$-methionine. Immunoprecipitation of the cell lysate was carried out with a monoclonal antibody (MAb) M2 specific to the flag (Statagene), which were fused to the C-terminus of each protein (Fig. 1). It was found 
that the full-length and deletion mutants of $\mathrm{Al}$ protein were expressed. The size of each protein appeared to correspond to its estimated molecular weight. These proteins are specific because the M2 antibody also detected HE-flag (Liao et al., 1995) in the positive control, but did not detect endogenous hnRNP Al protein or GFP in DE-GFP-transfected cell lysate.

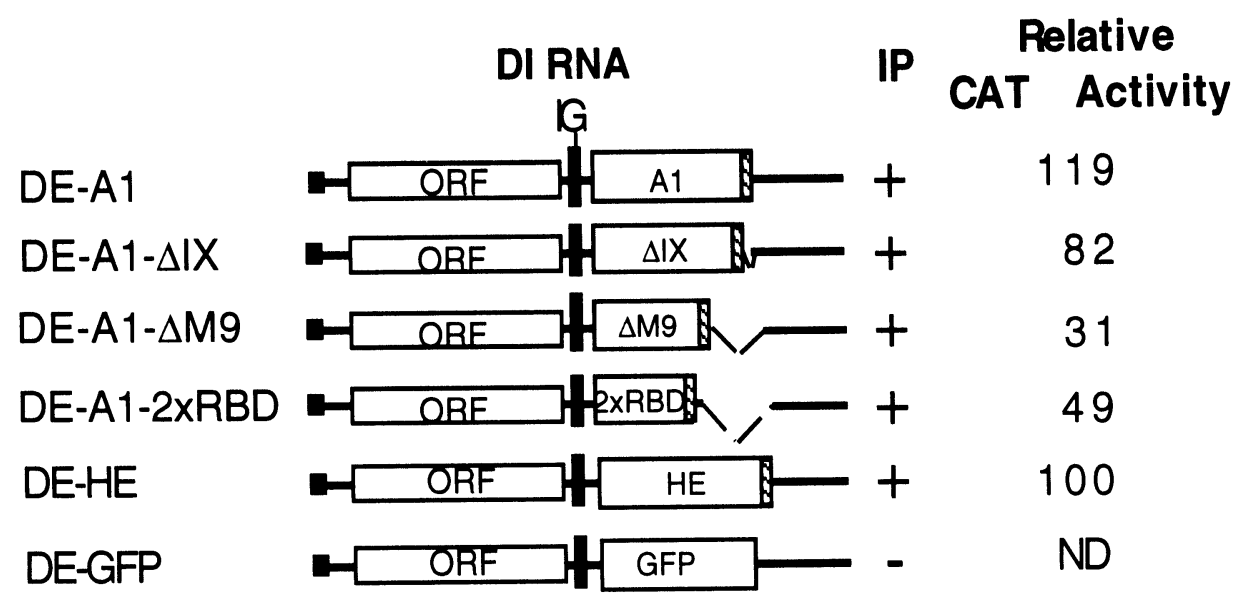

Figure 1. Effects of hnRNP-Al mutants on the expression of a reporter gene. The names and structures of individual DI RNAs are indicated on the left. IG, intergenic sequence. The flag at the $\mathrm{C}$-terminus is indicated by a striped box. IP, immunoprecipitation with anti-flag mAb. CAT activities in lysate from a $60 \mathrm{~mm}$-Petri dish $\left(5 \times 10^{6}\right.$ cells) were determined by a CAT assay. Data shown are the averages from three individual experiments. The absolute CAT activities (cpm) are converted to percentages relative to the CAT activities of MHV-infected and mock-transfected cell lysate, in which the CAT activity is set to $100 \%$. ND, not done.

\subsection{Dominant Negative Effects of Mutant hnRNP A1 Proteins on MHV RNA Synthesis}

We next determined the effects of Al deletion mutants on MHV RNA synthesis using the DI RNA CAT reporter system. Following the expression of these proteins, a CAT reporter DI RNA was super-transfected. Three hours after the reporter DI RNA transfection, cell lysate was extracted and CAT activities were determined. As shown in Fig. 1, CAT activities decreased by approximately 70,50 , and $20 \%$ in $\triangle \mathrm{M} 9-, 2 \mathrm{xBD}-$, and $\triangle \mathrm{IX}-$ expressing cells, respectively, as compared to those of the control (DE-HEflag). CAT activity was slightly higher in cells expressing the wild-type A1 (119\% of the control). We conclude that the deletion mutants of hnRNP Al exhibited dominant-negative effects on CAT expression. Because CAT activity in this DI system reflects the synthesis of a subgenomic DI RNA (Liao and Lai, 1994), this result suggests that these mutants have dominantnegative effects on MHV RNA synthesis. 
We then determined the effects of the mutant proteins on MHV RNA synthesis by directly measuring the amounts of de novo-synthesized viral RNAs. Cells were infected with MHV at an m.o.i. of 1, and transfected with DI RNAs containing the wild-type and mutant Al genes. Newlysynthesized intracellular RNAs were metabolically labeled with ${ }^{3} \mathrm{H}$-uridine in the presence of actinomycin D at $4 \mathrm{~h}$ post transfection for $4 \mathrm{~h}$, and quantified after TCA precipitation. Fig. 2 shows that the amounts of total TCA-precipitable materials in $\triangle \mathrm{IX}-, \Delta \mathrm{M} 9-, 2 \times \mathrm{RBD}$-expressing cells were approximately 10,70 , and $60 \%$ less than that in the control, in which the DE-CAT was expressed. Consistent with the CAT assay (Fig. 1), TCAprecipitable materials in wild-type Al-transfected cells were slightly more than that in the CAT control (Fig. 2). Because actinomycin D was added to inhibit host DNA-dependent RNA transcription $1 \mathrm{~h}$ prior to and throughout the labeling period, the radiolabeled materials most likely are de novosynthesized virus-specific RNAs. This interpretation is consistent with the result of Fig. 2, last column that only a background level of TCAprecipitable materials was detected from mock-infected and DE-CAT RNAtransfected cells, which also indicates that replication of DI RNAs depends on helper virus function. These results thus indicate that the mutants have dominant-negative effects on MHV RNA synthesis.

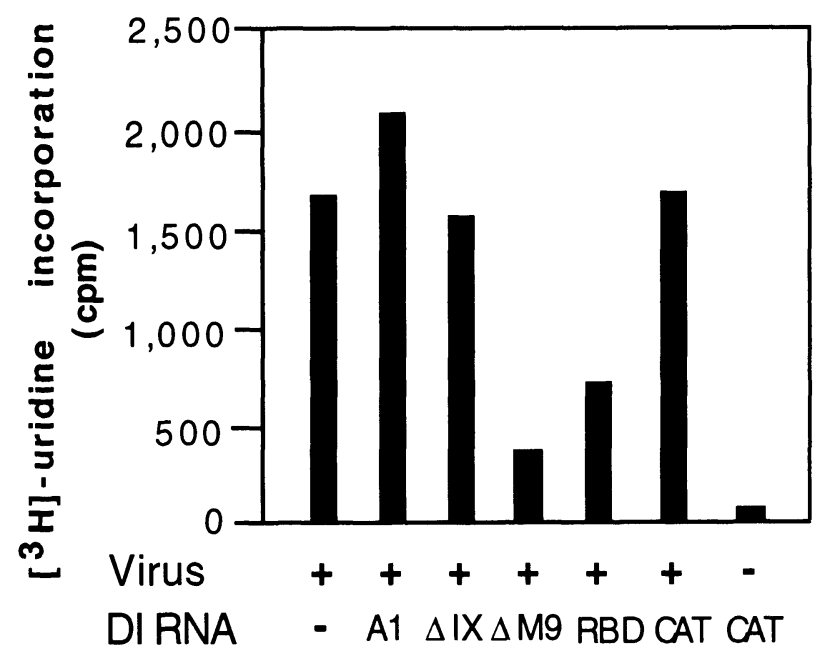

Figure 2. Effects of hnRNP-Al mutants on MHV RNA synthesis. DBT cells were infected without (-) or with (+) MHV JHM strain at a multiplicity of infection of 1 . At $1 \mathrm{~h}$ postinfection, cells were transfected without $(-)$ or with $(+) 5 \mu \mathrm{g}$ of DI RNAs containing various inserted genes as indicated. Actinomycin $D(10 \mu \mathrm{g} / \mathrm{ml})$ was added to the cells at $3 \mathrm{~h}$ posttransfection. One hour later, cells were labeled with $\left[{ }^{3} \mathrm{H}\right]$-uridine $(100 \mu \mathrm{Ci} / \mathrm{ml})$ for $3 \mathrm{~h}$. Intracellular RNAs were isolated. One $\mu 1$ of each RNA sample was precipitated by $5 \%$ TCA, and radioactivity in the precipitates were counted in a liquid scintillation counter. Data indicate the mean of a duplicate experiment for each sample. 


\subsection{Expression of Mutant hnRNP A1 Proteins by a Recombinant Vaccinia Virus and Their Effects on MHV RNA Synthesis}

We then used the recombinant vaccinia virus expression system to express mutant A1 proteins. Cells were infected with the recombinant vaccinia virus vTF7-3. At $1 \mathrm{~h}$ after vTF7-3 infection, $X b a$ I-linearized plasmid DNAs, which contain a wild-type or mutant hnRNP A1 genes behind the T7 promoter (Fig. 3), were transfected. Following incubation at $37^{\circ} \mathrm{C}$ for $10 \mathrm{~h}$, cells were superinfected with MHV JHM strain to provide helper function necessary for DI RNA replication. At $1 \mathrm{~h}$ after MHVinfection, in vitro-transcribed CAT-reporter DI RNAs were transfected. Cell extracts were isolated at $4 \mathrm{~h}$ after reporter-transfection, and CAT activities in the lysate were then determined. The CAT activity measured in this system reflects the total effects of replication and transcription of both DI RNA and helper virus. As shown in Fig. 3, CAT activities from $\Delta \mathrm{IX}-, \Delta \mathrm{M} 9-$ and 2xRBD-expressing cells were 16,71 , and $57 \%$, respectively, lower than that from the mock-transfected cells. In cells expressing the wild-type Al, the CAT activity was slightly higher. The CAT activity from GFP-expressing cells was also lower, for which the reason was unclear.

DNA transfected DNA

T 7 - A 1

T7 - A1- $\Delta I X$

T7-A1- $\Delta$ M9

T7 - A1 - 2 xRBD

T 7 - GFP
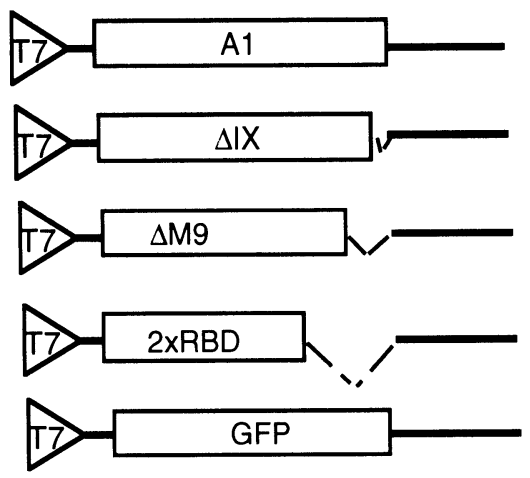

Relative CAT Activity

128

84

29

43

89

\section{Mock-t ransfect ed}

Figure 3. Effects of hnRNP-Al mutants expressed by a recombinant vaccinia virus on the expression of a reporter gene. The names and structures of individual DNA constructs are indicated on the left. CAT activities in lysate from a $60 \mathrm{~mm}$-Petri dish were determined by a CAT assay. Data shown are the mean value from a triplicate experiment. The absolute CAT activities (cpm) are converted to percentages relative to the CAT activities of vTF7-3-infected and mock-transfected cell lysate, in which the CAT activity is set to $100 \%$. 
In this study, we employed two different approaches to transiently express the wild-type and various deletion mutants of A1 protein in DBT cells and to determine their effects on MHV RNA synthesis. The advantage of using the DI RNA expression system is that it is devoid of potential interference by foreign genes. Its limitation is the dependence of DI gene expression on helper virus function. Therefore, on one hand, the more the helper virus replicates, the better the DI RNA gene expresses. On the other hand, when a large amount of helper virus RNA accumulate, dominantnegative effects may not be readily detected. This feature might have accounted for the low dominant-negative effect observed with the DI expression system (Fig. 1-2). The recombinant vaccinia virus expression system has the advantage in that the expression of the A1 mutants is independent of MHV function; once expressed, the effects of the mutants on MHV RNA synthesis can be observed early in MHV replication. Nevertheless, both approaches yielded similar results. Because both approaches express proteins transiently, the level of expression depends on the efficiency of transfection. We routinely achieved $\approx 10 \%$ transfection efficiency for RNA and much higher for DNA. Since the transfection efficiency was low, the number of cells doubly transfected with the expression DI and the reporter DI must have be extremely low. If so, one may wonder how we could observe any negative-effect of the mutant proteins. We speculate that, because most of the cells were fused to form polykaryons or even the whole monolayer was fused together at the time when the second DI (reporter) was transfected, the expressed proteins may have traveled between cells to exert their function. Indeed, when the GFP was expressed in the DI system, the fluorescence intensity was strong in a single cell prior to fusion and became weaker and more diffused when cells began to fuse (Zhang, unpublished observation).

We found that $\Delta \mathrm{M} 9$ mutant exhibited the strongest inhibitory effect on the reporter gene expression and MHV RNA synthesis, while the $\triangle \mathrm{IX}$ mutant had little such effect. Although both mutants contain RNA- and protein-binding activities (Zhang et al., 1999), $\Delta \mathrm{M} 9$ lacks the M9 domain, which is known to be involved in protein-transport between the nucleus and the cytoplasm, while the $\Delta \mathrm{IX}$ contains this domain. Thus, our data may suggest that the M9 domain may be involved in viral RNA synthesis. The lack of the functional M9 domain in the $\Delta \mathrm{M} 9$ mutant possibly leads to an inhibitory effect on RNA synthesis. However, this does explain why $2 \times R B D$, which has a larger deletion than $\triangle \mathrm{M} 9$, had lower negative-effects than $\Delta$ M9. Apparently, some sequences other than the M9 domain may be also involved in regulation. Nevertheless, our present data indicate that cellular protein hnRNP Al is functionally involved in coronavirus RNA synthesis. Whether such involvement is direct or indirect through other 
factors, and how it regulates viral RNA synthesis remain to be further investigated.

\section{ACKNOWLEDGEMENTS}

This work was supported by Research Project Grant RPG-98-090-01MBC from the American Cancer Society.

\section{REFERENCES}

Black, A.C., Luo, J., Chun, S., Baker, A., Faser, J.K., and Rosenblatt, J.D. 1996. Specific binding of polypyrimidine tract binding protein and hnRNP Al to HIV-1 CRS elements. Virus Genes 12: 275-285.

Black, A.C., Luo, J., Watanabe, C., Chun, S., Baker, A., Faser, J.K., Morgan, J.P., and Rosenblatt, J.D. 1995. Polypyrimidine tract-binding protein and heterogeneous nuclear ribonucleoprotein Al bind to human T-cell leukemia virus type 2 RNA regulatory elements. J. Virol. 69: 6852-6858.

Buvoli, M., Biamonti, G., Tsoulfas, P., Bassi, M.T., Ghetti, A., Riva, S., and Morandi C. 1988. cDNA cloning of human hnRNP protein Al reveals the existence of multiple mRNA isoforms. Nucl. Acids Res. 16: 3751-3770.

Buvoli, M., Cobianchi, F., and Riva, S. 1992. Interaction of hnRNP Al with snRNPs and premRNAs: evidence for a possible role of Al RNA annealing activity in the first steps of spliceosome assembly. Nucl. Acids Res. 20: 5017-5025.

Caputi, M., Mayeda, A., Krainer, A.R., and Zahler, A.M. 1999. HnRNPA/B proteins are required for inhibition of HIV-1 pre-mRNA splicing. EMBO J. 18:4060-4067.

Cartegni, L., Maconi, M., Morandi, E., Cobianchi, F., Riva, S., and Biamonti, G. 1996. hnRNP Al selectively interacts through its Gly-rich domain with different RNA-binding proteins. J. Mol. Biol. 259: 337-348.

Dreyfuss, G., Matunis, M.J., Pinol-Roma, S., and Burd, C.G. 1993. hnRNP proteins and the biogenesis of mRNA. Annu. Rev. Biochem. 62: 289-321.

Furuya, T., and Lai, M.M.C. 1993. Three different cellular proteins bind to the complementary sites on the 5'-end positive- and 3'-end negative-strands of mouse hepatitis virus RNA. J. Virol. 67: 7215-7222.

Hirano, N., Fujiwara, K., Hino, S., and Matsumoto, M. 1974. Replication and plaque formation of mouse hepatitis virus (MHV-2) in mouse cell line DBT culture. Arch. Gesamte Virusforsch. 44: 298-302.

Li, H.P., Zhang, X.M., Duncan, R., Comai, L., and Lai, M.M.C. 1997. Heterogeneous nuclear ribonucleoprotein $\mathrm{Al}$ binds to the transcription-regulatory region of mouse hepatitis virus RNA. Proc. Natl. Acad. Sci. USA 94: 9544-9549.

Li, H.P., Huang, P., Park, S., and Lai, M.M.C. 1999. Polypyrimidine tract-binding protein binds to the leader RNA of mouse hepatitis virus and serves as a regulator of viral transcription. J. Virol. 73:772-777.

Liao, C.L., and Lai, M.M.C. 1994. Requirement of the 5'-end genomic sequence as an upstream cis-acting element for coronavirus subgenomic mRNA transcription. J. Virol. 68: 4727-4737. 
Makino, S., and Lai, M.M.C. 1989. Evolution of the 5'-end of genomic RNA of murine coronaviruses during passages in vitro. Virology 169: 227-232.

Yu, W., and Leibowitz, J.L. 1995. Specific binding of host cellular proteins to multiple sites within the 3'-end of mouse hepatitis virus genomic RNA. J. Virol. 69:2016-2023.

Zhang, X.M., Liao, C.L., and Lai, M.M.C. 1994. Coronavirus leader RNA regulates and initiates subgenomic mRNA transcription both in trans and in cis. J. Virol. 68: 4738-4746.

Zhang, X.M., and Lai, M.M.C. 1995. Interactions between the cytoplasmic proteins and the intergenic (promoter) sequence of murine hepatitis virus RNAs: Correlation with the amounts of subgenomic mRNA transcribed. J. Virol. 69: 1637-1644.

Zhang, X.M., Li, H.P., Xue, W., and Lai, M.M.C. 1999. Formation of a ribonucleoprotein complex of mouse hepatitis virus involving heterogeneous nuclear ribonucleoprotein $\mathrm{Al}$ and transcription-regulatory elements of viral RNA. Virology 264:115-124. 